\title{
Case Report: 50 yr. Old Female with C/0 Abdominal Pain before One Day of Presentation
}

\author{
Sushila Ladumor ${ }^{1 *}$, Adham Darweesh ${ }^{2}$ and Hiba Esmayil ${ }^{3}$ \\ ${ }^{1}$ Consultant Radiologist, Clinical Imaging Department, Hamad Medical Corporation, HGH, Doha, Qatar, Assistant Professor in Clinical Radiology, Weil \\ Cornel Medical College, Qatar (WCMC-Q)
}

${ }^{2}$ Senior Consultant Radiologist, Clinical Imaging Department, Hamad Medical Corporation, HGH,Doha, Qatar, Assistant Professor in Clinical Radiology, Weil Cornel Medical College, Qatar (WCMC-Q)

${ }^{3}$ R1 Radiology Resident, Hamad Medical Corporation, HGH, Clinical Imaging, P.O. Box 3050, Qatar

Submission: September 15, 2017; Published: September 21, 2017

*Corresponding author: Sushila Ladumor B, Consultant Radiologist, Clinical Imaging Department, Hamad Medical Corporation, HGH, Doha, Qatar, Assistant Professor in Clinical Radiology, Weil Cornel Medical College, Doha, Qatar (WCMC-Q), E-mail: drsbladumor@yahoo.com

\begin{abstract}
Endometriosis is defined as the presence of endometrial tissue outside the uterine cavity. It is mainly found in the abdominal cavity, most commonly on the surface of the ovaries. It is an estrogen-dependent disease and is estimated to occur in $10 \%$ of the female population, mostly in women of reproductive age [1,2]. Endometriosis has been associated with pain and infertility. The gold standard for the diagnosis of endometriosis has been visual inspection by laparoscopy, preferably with histological confirmation [3]. Now US is also helpful as well as MRI is very important to characterize and help to differentiate with other cystic lesion in pelvis and as well as deep and superficial location of endometriosis and surrounding organ involvement. Endometriomas, also known as chocolate cysts or endometriotic cysts, are a localized form of endometriosis and are usually within the ovary. They are readily diagnosed on ultrasound, with most demonstrating classical radiographic
\end{abstract} features.

Keywords: Endometriosis; Endometriotic cysts; Ultrasound; Magnetic resonance imaging; Ovarian carcinoma; Ovary; Diagnosis; Laparoscopy Abbreviations: US: Ultrasound; MRI: Magnetic Resonance Imaging

\section{Case Report}

a. Chief Complaint: 50 yr old female with $\mathrm{C} / 0$ Abdominal pain since yesterday.

b. Past History: previous 4 normal deliveries, Hysterectomy before four years due to multiple uterine fibroids and menorrhagia. Presented with left lower abdominal pain, not associated with any Gastro Intestinal or Genito-Urinary Symptoms. Not known to have any Ovarian pathology before.

c. No prior Imaging available.

\section{A. Imaging}

i. Initial US recommended: (Figure 1)

ii. Diagnosis of US:

iii. US: Large abdominopelvic cystic mass from left side with internal thick septa and some heterogeneity with suggestion of small soft tissue component. No internal vascularity. Left ovary is not separately seen.

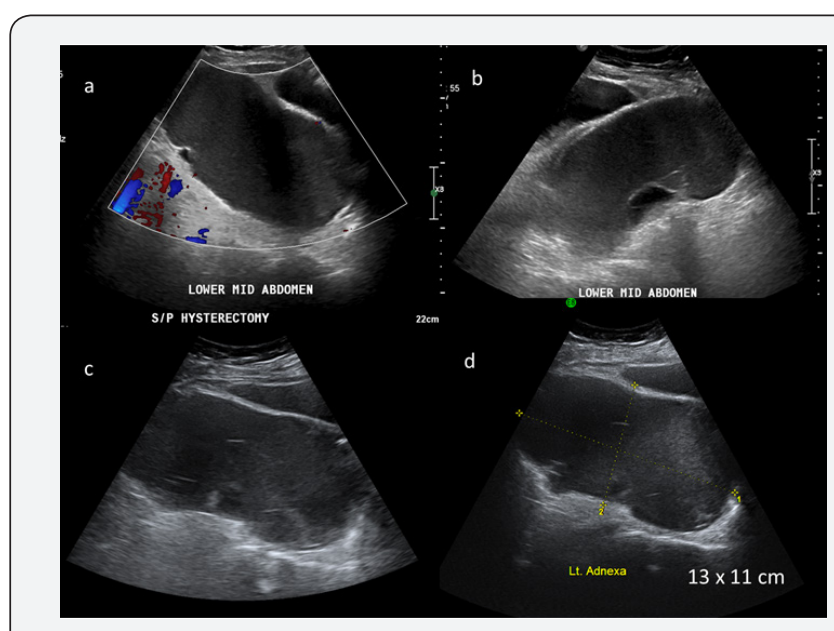

Figure 1: Abdominal US: Large complex predominantly cystic mass with internal septations and some heterogeneous component without definite solid component ( $a, b, c$ \& d) Color Doppler exam (a) show no internal vascularity. 
iv. D/D: Epithelial cystic tumor or endometrioma

v. Clinical laboratory correlation and MRI for better evaluation and characterization (Figures $2 \& 3$ ).

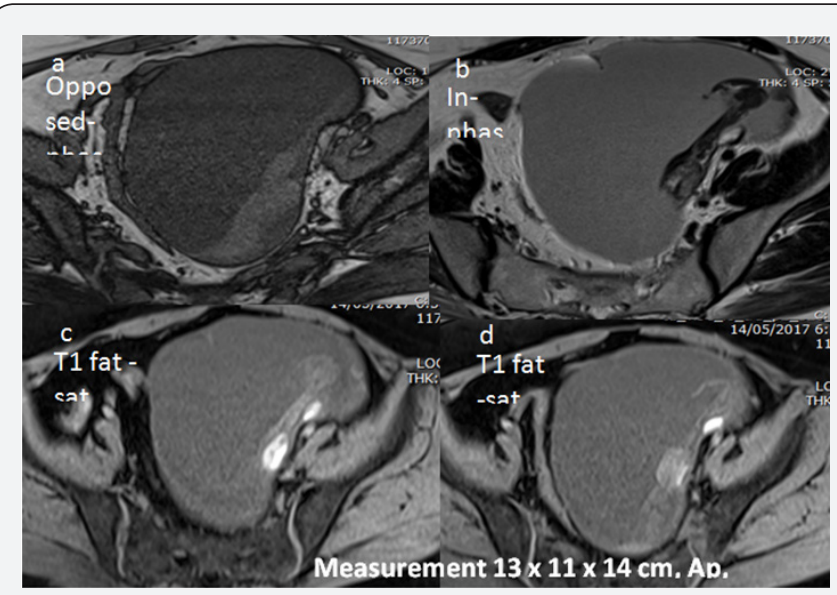

Figure 2: MRI requested for further work-up due to patient age and non-specific History

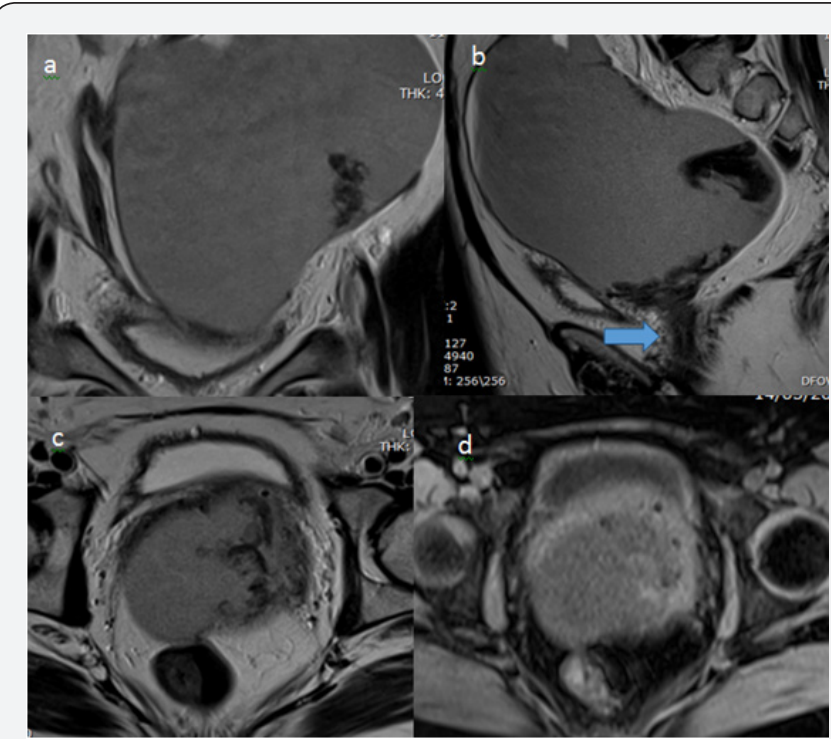

Figure 3: Axial images Opposed-phase (a) show no area of signal drop compare to in-phase (b) to suggest fatty component. In contrast there is area of dark signal in in-phase (b) compare to Opposed-phase (a) representing hemosiderin which shows slightly high signal in opposed phase (a) and higher signal in $\mathrm{T} 1$ fat sat. Foci of T1 fat sat (c \& d) bright signal representing hemorrhagic foci. Left ovary is not separately seen, this hemorrhagic foci could represent compressed left ovary with hemorrhagic follicles or hemorrhagic foci in cystic mass. Sigmoid colon is compressed and displaced to the right side (d).

\section{B. Epidemiology}

These occur predominantly in women of reproductive age.

\section{Clinical Symptoms}

The most common symptoms are dysmenorrhea, dyspareunia, pelvic pain, and infertility - although it may also be asymptomatic.

\section{Pathology:}

Endometriomas contains dark degenerated blood products following repeated cyclical hemorrhage. The cysts may be up to $20 \mathrm{~cm}$ in size although they are usually smaller $(2-5 \mathrm{~cm})$. Pathogenesis of endometriosis is complex and still debated [2]. Retrograde menstruation is neither sufficient nor necessary for the development of endometriosis. Two other theories postulate that endometriosis develops from the metaplasia of pelvic peritoneal tissue and from the transformation of circulating stem cells [2]. Several steps are required for the development of endometriosis [2]. First, endometrial glands and stroma must migrate beyond the uterus. Second, the ectopic endometrial cells must attach to the peritoneum, extend into the mesothelium, and grow.

T2 TSE (turbo spin-echo) axial a \&c) sagittal (d) T1 fat sat axial (d) demonstrates large pelvi-abdominal cystic lesion occupying anterior, anterior left lateral and mid pelvic space and extending into the pouch of Douglas showing low T1 and T2 signal with a fluid level and T2 dark signal areas along the dependent part mainly on left and posterior aspect. No appreciable soft tissue component. No area of diffusion restriction (Images are not shown). Urinary bladder in minimally distended and seen compressed anteriorly. No free fluid (Figure 4).

\section{E. MRI Impression}

Large abdominal pelvic cystic lesion with hemorrhagic component. Possibilities include peritoneal inclusion cyst/para ovarian cyst, endometriosis with turbid fluid and blood clots suggestion of endometriotic deposits within left ovary [4-6].

\section{a. Treatment}

i. Patient underwent post bilateral salpingoophrectomy + left cystectomy:

ii. Histopathology report

iii. Left and right ovaries and fallopian tubes + left ovarian cyst capsule, biopsy:

I. Ovarian endometriotic cyst.

II. Walthard cell rest, cystic.

III. Atrophic ovary.

IV. No malignancy.

\section{Discussion}

Endometriosis is defined as the presence of endometrial tissue outside the endometrium and myometrium. This condition is predominantly found in women of reproductive age and typically causes pelvic pain and infertility. Ovaries are one of the most common sites that endometriosis affects. One of the major treatment options is surgical removal of the ovaries; however, preservation of the reproductive function is desirable in most women with endometriosis. Incidence of clear cell and 
endometrioid adenocarcinomas are the malignancies reported in ovarian endometriosis seen as enhancing mural nodules in imaging [6].

A detailed history and physical examination, including speculum and bimanual examination, may be helpful in diagnosis. As an estrogen-dependent disease occurring in women with heavy menstrual bleeding, endometriosis has been suspected most commonly in women with menstrual-associated cyclic pain. Such cyclic pain is not pathognomonic for endometriosis, as women with fibroids and adenomyosis may also have dysmenorrhea. Furthermore, many patients with endometriosis have non-menstrual chronic pelvic pain, complaining of pain at other predictable times of their menstrual cycle, such as at ovulation. Patients may also have dyspareunia, bowel or bladder pain, or chronic fatigue [3]. No individual serum marker has yet been shown to be both sensitive and specific for diagnosis or monitoring of endometriosis. Further research is needed to establish the potential benefit of endometrial biopsy or panels of serum markers for the noninvasive diagnosis of endometriosis. Attempts to diagnose endometriosis at unusual sites remain investigational [3].

\section{Where Endometriomas occurs?}

Typical locations include:

\section{A. Superficial endometriosis}

i. In superficial endometriosis - also known as Sampson's syndrome - superficial plaques are scattered across the peritoneum, ovaries and uterine ligaments.

ii. These patients tend to have minor symptoms and usually also less structural changes in the pelvis.

\section{B. Deep pelvic endometriosis}

i. In deep pelvic endometriosis - also called Cullen's syndrome - there is sub peritoneal infiltration of endometrial deposits.

ii. The symptoms are more severe and related to the localization and depth of invasion in Cul-de-sac, Uterus, Bowel involvement, Urinary Bladder involvement, Adhesions.

iii. Main Location of Endometriosis:

a. Ovaries: $\sim 75 \%$

b. Anterior/posterior cul-de-sac: $\sim 70 \%$

c. Posterior broad ligament: $\sim 50 \%$

d. Uterosacral ligaments: $\sim 35 \%$

e. Uterus: $\sim 10 \%$

f. Colon and Urinary bladder: $\sim 5 \%$

\section{Radiographic features}

a. Plain radiograph: Not usually helpful in diagnosis; 10\% of endometriomas can calcify. b. Ultrasound: The appearances of endometriomas can be quite variable. It can appear asanunilocular cyst with acoustic enhancement with diffuse homogeneous ground-glass echoes as a result of the hemorrhagic debris. This appearance usually seen up-to $50 \%$ of cases.

Less typical features include:

i. Multiplelocules ( $\sim 85 \%$ will have $<5$ locules $)$

ii. Hyperechoic wall foci (present in 35\%)

iii. Cystic-solid lesion $(\sim 15 \%)$ or purely solid lesion $(1 \%)$

iv. Anechoic cysts (rare; 2\%)

\section{MRI}

Signal characteristics vary according to the age of any complicating hemorrhage:

i. T1 Weighted Sequences

ii. Typically, lesions appear hyper intense while acute hemorrhage occasionally appears hypo intense. Endometriomas with high T1 signal characteristically do not show loss of signal on T1 fat suppressed sequence, which is important to differentiate from mature cystic teratoma of the ovary. Dual Gradient-Echo InPhase and Opposed-Phase imaging also helpful in detection of intra cellular lipid which is seen in ovarian teratomas.

iii. T2Weighted Sequences

iv. Typicallyhypo intense owing to the presence of deoxyhemoglobin and methaemoglobin (shading sign), which is very suggestive of endometrioma. T2 dark spot sign is specific for chronic hemorrhage and is helpful in diagnosing endometriomas. Old haemorrhage occasionally appears hyper intense

d. DWI: Variable restricted diffusion. It must be remembered that benignendometriomas, like many pelvic malignancies, may exhibit restricted diffusion.

e. T1C+: May have wall enhancement and presence of an enhancing mural nodule may suggest malignant transformation

\section{Differential diagnosis}

The differential diagnosis of endometrial cysts includes: hemorrhagic functional cysts (brighter on T2-weighted images absence of the "shading sign") [5], fibrothecoma ( Low signal in T1 and T2 weighted sequence with some delayed postcontrast enhancement), cystic mature teratoma (will show fat suppression on fat suppressed sequences on MRI and drop of signal in opposed phase compared to in-Phase), cystic ovarian neoplasm ( Homogenous signal in benign epithelial neoplasm and variable small to large soft tissue component which shows restricted diffusion and post-contrast enhancement in malignant epithelial neoplasm with ancillary Imaging findings) and ovarian abscess ( shows central restricted diffusion with thick peripheral wall enhancement along with clinical presentation). 


\section{Conclusion}

Studies have shown that experienced clinicians can predict the presence of endometriosis based on history and physical examination in $80 \%$ of cases. Transvaginal ultrasound, MRI or transrectal ultrasound can help visualize endometriomas and deeply infiltrating endometriosis. Transvaginal ultrasound is likely superior to transrectal ultrasound in the majority of cases. MRI is particularly helpful in ultrasonographicallyindeterminate pelvic masses and diagnosing endometriosis of the ureters, bladder, and rectosigmoid.

\section{References}

1. Evan S Siegelman, Edward R Oliver (2017) MR Imaging of Endometriosis: Ten Imaging Pearls 1.
2. Jensen JR, Coddington CC (2010) Evolving spectrum: the pathogenesis of endometriosis. Clin Obstet Gynecol 53(2): 379-388.

3. Hsu AL, Khachikyan I, Stratton P (2010) Invasive and noninvasive methods for the diagnosis of endometriosis. Clin Obstet Gynecol 53(2): 413-419.

4. Glastonbury CM (2002) The shading sign. Radiology 224 (1): 199-201.

5. Woodward PJ, Sohaey R, Mezzetti TP (2010) Endometriosis: radiologicpathologic correlation. RadioGraphics 21(1): 193-216; questionnaire 288-294.

6. Michael T Corwin, Eugenio O Gerscovich, Ramit Lamba, Machelle Wilson, John P McGahan (2016) Differentiation of Ovarian Endometriomas from Hemorrhagic Cysts at MR Imaging: Utility of the T2 Dark Spot Sign, Radiology.

\section{Your next submission with Juniper Publishers will reach you the below assets}

- Quality Editorial service

- Swift Peer Review

- Reprints availability

- E-prints Service

- Manuscript Podcast for convenient understanding

- Global attainment for your research

- Manuscript accessibility in different formats

( Pdf, E-pub, Full Text, Audio)

- Unceasing customer service

Track the below URL for one-step submission https://juniperpublishers.com/online-submission.php 